\title{
Attention in the Acquisition and Expression of Automaticity
}

\author{
Gordon D. Logan, Stanley E. Taylor, and Joseph L. Etherton \\ University of Illinois
}

\begin{abstract}
The instance theory of automaticity claims that people learn what they attend to and express what they learned in transfer if they attend to the same things in the same way. These hypotheses were tested in 8 category search experiments in which target position was cued by color (red or green). The main question was whether target color would be encoded in training and retrieved in transfer. After training, recognition memory for target color was above chance, which suggests that color was encoded. However, category search performance was not affected by changing target color unless color was reported explicitly during training and transfer, which suggests that color was not always retrieved. The results are consistent with the instance theory. The distinction between encoding and retrieval is important in understanding the acquisition and expression of automaticity. Automatic performance emphasizes speed and so may not be sensitive to things that are retrieved slowly.
\end{abstract}

This article concerns the role of attention in the acquisition and expression of automaticity. This is an important issue in the automaticity literature, especially from the perspective of theories that assume that automatic performance is based on retrieval of past solutions from memory (e.g., Logan, 1988, 1990). According to those theories, attention determines what is learned during practice and it determines what is retrieved from memory to support performance after some degree of automaticity has been achieved. The role of attention in encoding and retrieval is an important issue in the broader literature on learning and memory (e.g., Cohen, Ivry, \& Keele, 1990; Craik \& Lockhart, 1972; Kirsner \& Dunn, 1985; Morris, Bransford, \& Franks, 1977; Nissen \& Bullemer, 1987; Roediger, 1990; Stadler, 1995), so the data should have relevance beyond the automaticity literature.

\section{The Attention Hypothesis}

The present experiments were designed to investigate the attention hypothesis, which was derived from the instance theory of automaticity (Logan, 1988, 1990; Logan \& Etherton, 1994). The attention hypothesis is an attempt to explain what is learned during automatization, to explain what gets into an instance. The attention hypothesis was derived from the obligatory encoding assumption of the instance theory, which claims that learning is a side effect of attending: People will learn about the things they attend to and they will not learn

Gordon D. Logan, Stanley E. Taylor, and Joseph L. Etherton, Department of Psychology, University of Illinois at Urbana-Champaign.

Experiments 1 and 3 were part of Joseph L. Etherton's master's thesis. This research was supported by National Science Foundation Grants BNS 88-11026 and BNS 91-09856. We are grateful to Connie Boronat, Jane Zbrodoff, Richard Ivry, and Nelson Cowan for helpful comments on the article and to Julie Delheimer for testing some of the subjects and for analyzing the data.

Correspondence concerning this article should be addressed to Gordon D. Logan, Department of Psychology, University of Illinois, 603 East Daniel Street, Champaign, Illinois 61820. Electronic mail may be sent via Internet to glogan@s.psych.uiuc.edu. much about the things they do not attend to (Logan, 1988, 1990; Logan \& Etherton, 1994; see also Barsalou, 1995).

The present article extends the attention hypothesis beyond encoding to retrieval. Attention determines what is taken out of memory at retrieval time as well as what gets into memory at encoding. Attention determines the retrieval cues that drive the retrieval process so that things related to the current object of attention are retrieved. Things not related to it are not retrieved. This extension of the attention hypothesis is derived from the obligatory retrieval assumption of the instance theory of automaticity, which claims that retrieval is a side effect of attending (Logan, 1988, 1990). The idea has precedents in the memory literature, such as Tulving and Thompson's (1973) encoding specificity principle and Morris et al.'s (1977) transferappropriate processing hypothesis. It is closely related to more recent process-oriented interpretations of associations and dissociations between implicit and explicit memory (e.g., Hintzman, 1990; Jacoby \& Brooks, 1984; Roediger, 1990). The general point of these analyses is that memory performance depends on both encoding and retrieval. Everything that is retrieved must have been encoded, but everything that is encoded is not necessarily retrieved. A given retrieval task may not access everything that was learned. Our goal was to apply these ideas to automaticity.

\section{Automaticity and Memory}

The main focus of the present article is on automaticity and the memory traces that support automatic performance. The instance theory assumes that automaticity is a memory phenomenon, like recognition, recall, and the various tests of implicit memory, and like the other memory phenomena, automaticity imposes special constraints on the retrieval process. The instance theory assumes that performance depends on the outcome of a race between a general algorithm for performing the task and a memory process that retrieves past solutions. Performance is automatic when memory wins the race (Logan, $1988,1990,1992 b)$. The race with the algorithm - and, indeed, the race with other traces in memory-imposes constraints on the retrieval process, emphasizing speed and ease of access. 
Thus, the memory traces that support automatic performance may be different from those that support performance on memory tasks that place less emphasis on speed and ease of access. Traces that are not accessed quickly and easily may contribute to other retrieval tasks, such as recognition memory, perceptual identification, or fragment completion, even though they do not contribute much to automatic performance.

These considerations make it important to distinguish between encoding and retrieval when asking what is in an instance. The instances that are retrieved are not exactly the same as the instances that were encoded. Retrieved instances are a subset of the encoded instances. Automatic performance depends on both encoding and retrieval, so evidence that some aspect of a stimulus is important in automatic performance suggests that that aspect was encoded in the instance. However, evidence that some aspect of a stimulus is not important in automatic performance does not mean that that aspect was not encoded. It may be available to some other retrieval task. What is learned during automatization and what is expressed during automatic performance are logically separate questions.

\section{Previous Investigations of the Attention Hypothesis}

The strength of the attention hypothesis is that it can be tested. Attention can be manipulated as an independent variable and its effects can be observed on immediate performance as well as on subsequent performance on memory tasks. The effects on immediate performance provide a manipulation check that allows attention to be defined operationally without circularity. There is a large literature on the effects of attention on immediate performance that can be exploited for this purpose (e.g., Broadbent, 1958; Kahneman, 1973). Once attention has been defined in immediate performance, its subsequent effects on memory performance can be predicted, and failures of those predictions can disconfirm the attention hypothesis.

Much of the previous research on the attention hypothesis used a distinction, proposed by Treisman (1969), between four different kinds of attention: input selection, analyzer selection, target selection, and response or output selection. The different kinds of attention are defined in terms of analyses applied to stimuli. Input selection involves choosing which stimulus or set of stimuli to analyze, analyzer selection involves choosing which kind of analysis to perform on the (input) selected stimuli, target selection involves choosing a course of action depending on the result of an analysis, and output or response selection involves choosing an overt response to execute as a result of the analysis (Treisman, 1969).

\section{Input Selection}

Logan and Etherton (1994) investigated input selection in a category search task. They presented people with two words and asked them to decide whether one of the words was a member of a target category (e.g., metals). They manipulated input selection by providing (focused attention) or withholding (divided attention) cues that indicated which of the two words would be the target. Their purpose was to test whether people would learn more about the distractors in the dividedattention condition, in which both words were attended, than in the focused-attention condition, in which only targets were attended. They paired particular targets with particular distractors consistently throughout training and changed the pairing at transfer. Thus, if Steel was presented with Canada when it first appeared, it was presented with Canada on all subsequent appearances in the training session(s). At transfer, it appeared with a different distractor (e.g., France). The attention hypothesis predicts that people should be sensitive to the pairing of targets and distractors in the divided-attention condition, because they attend to both words, but not in the focusedattention condition, because they attend only to targets.

The results were consistent with the attention hypothesis. Changing the pairing at transfer slowed categorical decisions by $99 \mathrm{~ms}$ in divided-attention conditions and $9 \mathrm{~ms}$ in the focused-attention conditions. These results were replicated with high (64 repetitions over four sessions) and low (16 repetitions in one session) levels of practice and were corroborated by differences in recall of distractors in a surprise recall test $(36.1 \%$ of the distractors were recalled in divided attention and $24.1 \%$ in focused attention). Input selection appears to determine what is learned during automatization and expressed in automatic performance.

\section{Analyzer Selection}

Logan $(1988,1990)$ examined analyzer selection by presenting words, pronounceable nonwords, and unpronounceable nonwords and varying the analysis performed on them. One analysis involved lexical decisions, in which people said yes to words and no to pronounceable and unpronounceable nonwords. The other analysis involved pronounceability decisions, in which people said yes to words and pronounceable nonwords and no to unpronounceable nonwords. Subjects either performed one kind of analysis consistently (half doing lexical decisions and half doing pronounceability decisions) or alternated between them. The letter strings were presented up to 16 times to both groups of subjects, and benefits from repetition were calculated over practice. The attention hypothesis predicts greater repetition benefit for subjects who performed consistent analyses than for subjects who alternated between analyses because the former subjects would have more relevant traces to retrieve than the latter. The results confirmed this prediction. Thus, analyzer selection also appears to determine what is learned during automatization and what is expressed in automatic performance.

\section{Output Selection}

Logan (1990) investigated output or response selection in a lexical-decision task. Subjects made lexical decisions on the same words and nonwords for several repetitions. In one experiment, subjects were transferred to a different mapping of response categories onto response keys (e.g., those who pressed the right key for words and the left key for nonwords now did the opposite). In another experiment, one group maintained the same mapping of response categories onto response keys throughout practice, and another group alter- 
nated between mappings (e.g., pressing the right key for words in one block and the left key for words in the next). There was no effect of changing response mapping in either experiment. These experiments suggest that the physical response that is executed to report the decision is not an important part of the memory trace that supports automatic performance. The results corroborate findings in letter search experiments, in which the physical response was changed from trial to trial with no effect on performance, provided that the mapping of stimuli to response categories remained consistent (Fisk \& Schneider, 1984; Shiffrin \& Schneider, 1977, Experiment 3). Together, these results suggest that output or response selection does not exert strong constraints on the development and expression of automaticity.

\section{Target Selection: The Present Experiments}

In the present experiments we extended the investigation of the attention hypothesis to target selection, the fourth and final type of selective attention distinguished by Treisman (1969). Target selection occurs when people choose among courses of action depending on the outcome of an analysis, doing one thing if the input matches the target characteristics and another if it does not. Target selection is involved in search tasks, in which subjects respond if they detect a target and continue searching if they do not. It is also involved in filtering tasks, in which subjects process the input further if it possesses the target feature but stop if it does not (Kahneman, Treisman, \& Burkell, 1983; Treisman, Kahneman, \& Burkell, 1983). Focused-attention experiments involve the filtering type of target selection. In Logan and Etherton's (1994) focusedattention experiments, for example, subjects saw a green word and a white word and were instructed to assess category membership only for the green word. The analysis of green and the subsequent choice of a course of action (to assess or not to assess category membership) is an example of target selection. According to the attention hypothesis, when subjects select a target in a filtering task (a task that requires focused attention), they must attend to the value of the target feature, so that feature should be stored in memory as part of the instance created on that trial.

In the present experiments we investigated the attention hypothesis, applied to target selection, by modifying Logan and Etherton's (1994) focused-attention task such that the target color was either red or green. Subjects performed a category search task, deciding whether two-word displays contained a member of a target category (e.g., metals). One of the words in each pair was red or green, and the other word was white. Subjects were instructed to assess category membership of the colored word (red or green) and to ignore the white word. To select the appropriate word, subjects would have to test for color and assess category membership only if the word was red or green. Under these conditions, colors should be associated with target words. If Steel appeared in red, it should be associated with red. The pairing of colors and words was consistent throughout the 16-block training phase of the experiment. If Steel appeared in red in the first block, it would appear in red in all 16 training blocks.

Learning of specific color-word pairs was assessed with two retrieval tasks administered in a transfer block: a recognition memory task (Experiments 1 and 2) and the same categorization task used in training (Experiments $3-8$ ). The recognition memory task presented words in the same color or in the opposite color they appeared in during training and subjects were asked to judge whether the word appeared in that color during training. The task addressed color-word pairing directly and explicitly, so we expected it to be sensitive to the presence of color-word associations in the memory trace. Moreover, the recognition task did not require speeded responses, so it should be sensitive to traces of color-word associations, even if they were retrieved slowly.

The categorization task presented words in the opposite color (Experiments 3 and 4) or in the same and in the opposite color (Experiments 5-8) they appeared in during training. Subjects were required to make the same categorical decisions they made throughout training (for the most part) but with a greater degree of automaticity. The purpose was to assess the importance of color-word associations in the traces that supported automatic performance. The categorization task addressed color-word associations indirectly. We expected facilitation if color-word pairing was the same and interference if it was different. Moreover, the categorization task required speeded responses, so it should be less sensitive than the recognition memory task to traces that were retrieved slowly.

\section{Separating Encoding and Retrieval Processes}

According to the attention hypothesis, attention determines what is encoded and what is retrieved, but not everything that is encoded is necessarily retrieved. To separate encoding from retrieval, we varied the response requirements in training and transfer. Subjects were either required or not required to report the color of the targets. Those who were not required to report target color simply pressed one key if the colored word was a member of the target category and another key if it was not, as in Logan and Etherton's (1994) experiments. Subjects who were required to report target color pressed one of three keys. If the colored word was a member of the target category and it was colored red, they pressed one key. If it was a member of the target category and it was colored green, they pressed a second key. If it was not a member of the target category, they pressed the third key.

We assumed that explicitly requiring subjects to report target color would ensure that target color was encoded in the memory trace. We assumed further that explicitly requiring subjects to report target color would cause target color to be retrieved if it was available in the memory trace. We used these report conditions to interpret the results we obtained in the experiments (with the more usual procedure) in which target color was not reported explicitly. Subjects may not encode target color if they do not have to report it explicitly, or they may encode it but not retrieve it if they do not have to report it explicitly.

\section{Automaticity}

Automaticity was produced by training under consistentmapping conditions (Shiffrin \& Schneider, 1977). Subjects 
searched for members of a target category, mapping was consistent in that the target category was the same throughout training, and the specific examples presented were the same throughout training. Practice with consistent mapping produces the changes associated with automatization: a reduction in reaction time, a reduction in load effects, and a reduction in dual-task interference (Logan \& Etherton, 1994).

There were 16 blocks of training trials, and each example of the target category was presented once per block. This may seem like a small amount of practice for an automaticity experiment, but we have shown repeatedly that extensive training is not necessary to produce the qualitative changes associated with automatization (Lassaline \& Logan, 1993; Logan, 1988, 1990; Logan \& Klapp, 1991). Logan and Etherton (1994) compared large (64-block) and small (16-block) amounts of practice on the same category search task used in the present experiments and found the same qualitative effects at both levels of practice. There was a power function reduction in reaction time, a reduction in load effects, and a reduction in dual-task interference at both levels of practice. Moreover, the transfer effects (costs in divided-attention and dual-task conditions and lack of cost in focused-attention conditions) were the same at the two levels of practice. Because their experiments were so similar to the present ones, in which the same task, stimuli, display apparatus, and subject population were used, we did not test for automatization as rigorously as they did. We defined automatization in terms of a power function reduction in reaction time. We did not test for a reduction in load effects with practice or a reduction in dual-task interference.

\section{The Present Experiments}

We conducted eight experiments, divided into three sets. In the first set (Experiments 1 and 2) we tested recognition memory for target colors after 16 blocks of training. Subjects reported target color in training in one experiment but not in the other. In the second set (Experiments 3-6) we transferred subjects to a variation of the category search task that we used in training. The experiments were a factorial combination of color report or no color report in training and color report or no color report in transfer. In the third set (Experiments 7 and 8) we examined ancillary hypotheses about the transfer effects seen in the second set, requiring color report in training and in transfer but changing the response keys between training and transfer.

\section{Experiment 1: No Report in Training and Recognition Test at Transfer}

In Experiment 1 we trained subjects on the focusedattention categorization task and transferred them to a recognition memory task that required them to discriminate words presented in the same color they appeared in during training from words presented in the opposite color. The purpose was to document the development of automaticity (in the form of a power function speedup) in this task and to test for the presence of color-word associations in a retrieval task that was designed to be sensitive to them.
During training, subjects reported target presence and absence without explicitly reporting target color. After training, they were transferred to a recognition memory task in which pairs of words were presented, one colored red or green and the other colored white, just as in training. The recognition memory task required them to report whether the colored word had appeared in that color throughout training, regardless of its status as a member of the target category. If subjects associated words with colors during training, they should perform above chance on the recognition test. If they did not associate words with colors in training, their recognition performance should be at chance levels.

\section{Method}

Subjects. The subjects were 32 volunteers. Eighteen of them were sampled from the introductory psychology subject pool. They received course credit for participation. Fourteen subjects were recruited with sign-up sheets posted in the psychology building and were paid $\$ 4$ for participating. Each subject was screened for red-green color blindness with the Ishihara (1987) test. ${ }^{1}$

Apparatus and stimuli. The stimuli were 64 words used by Logan and Etherton (1994). They were drawn from four categories in the Battig and Montague (1969) norms, with 16 words in each category. The categories were metals, countries, vegetables, and articles of fumiture. The words are presented in the Appendix. The categories were matched with respect to frequency of mention in the Battig and Montague (1969) norms, prototypicality in the Uyeda and Mandler (1980) norms, word frequency in the Kucera and Francis (1967) norms, and word length in letters. Summary statistics for these measures are presented in Table 1 . The only significant differences between categories in these measures were in word frequency, in which the difference between the highest and lowest frequency categories was significant. Word frequency is not an important variable in category verification tasks (Balota \& Chumbley, 1984), and we counterbalanced assignment of categories to experimental conditions.

The words were displayed on Amdek model 722 color monitors driven by IBM PC XT and AT computers. There were four computers, each facing a different wall of a large room so that several subjects could be tested at the same time without distracting each other.

Two words were displayed on each trial, one above the other. The words were presented in the center of the screen but were left justified. Their initial letters appeared in column 33 of row 12 and row 13 on the standard $80 \times 24$ IBM text screen. The words were written in lowercase with the first letter capitalized. Viewed at a distance of 60 $\mathrm{cm}$, single words subtended $0.48^{\circ}$ of visual angle in height and a minimum of $0.76^{\circ}$ and a maximum of $2.29^{\circ}$ in length. The two-word displays subtended $1.14^{\circ}$ of visual angle vertically.

Each word pair was preceded by a fixation and warning display. It consisted of two lines of seven dashes centered in the screen. One line of dashes appeared one line above the top word (i.e., row 11, columns 32-38), and one appeared one line below the bottom word (i.e., row 14 , columns 32-38). Viewed at a distance of $60 \mathrm{~cm}$, the fixation and warning display subtended $1.62^{\circ}$ of visual angle horizontally and $1.72^{\circ}$ vertically.

\footnotetext{
${ }^{1}$ Paid subjects may behave differently than volunteers, so we compared their performance in training and transfer by repeating the ANOVAs with subject type (paid vs. volunteer) as a factor. Subject type produced no main effects or interactions in the training ANOVAs on reaction time and error rates and no main effects or interactions in the transfer ANOVAs on hits minus false alarms and $d^{\prime}$ scores.
} 
Table 1

Means and Standard Deviations of Measures of Word Frequency, Frequency of Mention, Prototypicality, and Word Length for the 16 Words in Each Category

\begin{tabular}{|c|c|c|c|c|c|c|c|c|}
\hline \multirow[b]{2}{*}{ Measure } & \multicolumn{2}{|c|}{ Metals } & \multicolumn{2}{|c|}{ Countries } & \multicolumn{2}{|c|}{ Vegetables } & \multicolumn{2}{|c|}{ Furniture } \\
\hline & $\bar{M}$ & $S D$ & $\bar{M}$ & $S D$ & $M$ & $\overline{S D}$ & $M$ & $\overline{S D}$ \\
\hline Word frequency & 18.90 & 15.20 & 51.40 & 60.90 & 8.80 & 11.70 & 47.80 & 64.50 \\
\hline Frequency of mention ${ }^{b}$ & 160 & 110 & 145 & 99 & 161 & 91 & 153 & 144 \\
\hline Prototypicalityc & 2.28 & 0.97 & 2.27 & 0.36 & 2.52 & 0.53 & 2.43 & 0.83 \\
\hline Word length & 5.81 & 1.80 & 6.56 & 1.55 & 6.63 & 1.93 & 5.63 & 1.89 \\
\hline
\end{tabular}

(1980).

Each trial began with the fixation and warning display exposed for $500 \mathrm{~ms}$. That display was extinguished and immediately replaced by a word pair, which was exposed for $1,000 \mathrm{~ms}$ in the training block and $3,000 \mathrm{~ms}$ in the recognition test in the transfer block. Then the screen went blank for $2,000 \mathrm{~ms}$ until the next trial began. Presentation time was extended in the recognition test to encourage slow responses and to maximize sensitivity to traces that were not retrieved quickly.

During training, responses were made by pressing the $>$ and / keys on the bottom row of the standard QWERTY keyboard with the index and middle fingers of the right hand. During the recognition test at transfer, responses were made by pressing the $z$ and $x$ keys with the index and middle fingers of the left hand.

Procedure. The experiment was organized in blocks of 32 trials, in which the 64 words were paired and each pair was presented once. Subjects were tested in Logan and Etherton's (1994) consistentpairing condition: The words were paired randomly at the beginning of the experiment, and the pairing remained the same throughout training and transfer, although the order in which the pairs were presented was randomized each block. A different random pairing was constructed for each subject.

There were two basic trial types, target present and target absent, and 16 of each type were presented in each block. On target-present trials, one word was selected from the target category, and one word was selected from one of two distractor categories. On target-absent trials, one word was selected from a fourth, nontarget category, and the other was selected from one of two distractor categories. Each of the four categories was used equally often as targets, nontargets, and each of the two distractor categories. The categories were assigned to these roles with a balanced Latin square.

Targets appeared equally often in the top and bottom positions in the display, as did nontargets and members of each of the two distractor categories. However, specific words were presented consistently in one position or the other. For example, if Canada was on top and Steel was on the bottom in the first block, they remained in those positions throughout training and transfer. Etherton (1992) investigated the effects of location consistency and found that they were minimal.

Half of the targets were colored red (IBM 12), and half were colored green (IBM 10). Half of the nontargets were colored red, and half were colored green as well. All of the distractors (one per display) were colored white (IBM 15). Thus, each display contained one white word and one red or green word. Specific words were presented consistently in one color or the other. For example, if Canada was a target and colored red in the first block, it appeared in red in each of the 16 training blocks. If France appeared in green in the first block, it appeared in green throughout training.

After the 16 training blocks, there was a recognition test that involved a single block of 32 trials in which half of the words (eight targets and eight nontargets) were colored the same as they were in training, and half of the words (eight targets and eight nontargets) were colored oppositely. We used this procedure instead of presenting each word twice (once in each color) so that subjects would not confuse prior presentations in the transfer block with prior presentations in the training block. For example, subjects who saw Canada in red throughout training and in green in the first part of the transfer block might be confused when it appeared in red later in transfer. In our procedure, a word was either consistent with all previous presentations or inconsistent with them. It was never consistent with some and inconsistent with others.

Subjects were given written instructions that described the task, told the name of their target category, and told which keys to press to indicate target presence and absence. They were not told about the recognition test until the end of training. Half of the subjects were told to indicate target presence with the index finger of their right hand and target absence with the middle finger, and half were told the opposite. All subjects were told to rest their index and middle fingers of their right hand lightly on the keys throughout training. None were told about the number or the nature of the nontarget and distractor categories. They were told to pay attention to the colored word because the target would always be colored red or green and if the red or green word was not a target, there would be no target in the display. After they read the instructions, the experimenter summarized them and answered questions. Then the experiment began. Subjects were allowed brief rests every 128 trials (four blocks). The last rest was just before the transfer trials.

The transfer block was announced by an instruction, presented on the computer screen, that told subjects to call the experimenter for further instructions. The experimenter gave them written instructions that indicated they would see the training words once again, but half of them would be colored in the same way they were in training, and half would be colored differently. Subjects were instructed to press one key if they thought that the colored word was colored the same as it was in training and to press another key if they thought it was colored differently. They were told to take their time in making their recognition decision and that accuracy was more important than speed. This instruction diminished the likelihood that subjects would respond on the basis of the most quickly retrieved traces and allowed us to determine whether color information is available in memory but not as soon as other information.

\section{Results}

Training. The mean reaction times and percentage of error scores for target-present and target-absent responses in the 16 training blocks are presented in Figure 1. Reaction times were faster for target-present than for target-absent responses, reflecting a tendency for yes responses to be faster than no responses. Error rate was higher for target-present responses, 


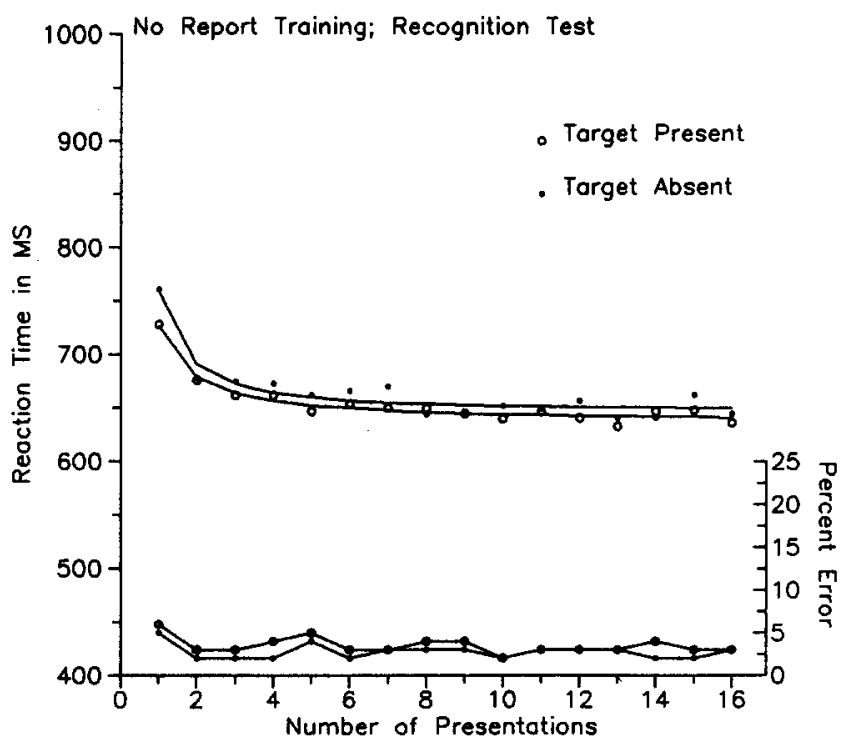

Figure 1. Mean reaction times (top two lines, left-hand $y$ axis) and error rates (bottom two lines, right-hand $y$ axis) for target-present (open circles) and target-absent (filled circles) responses from the training phase of Experiment 1 as a function of number of presentations (for the reaction times, the lines represent the best fitting power function, and the circles represent the observed data).

suggesting that people were more likely to say no than to say yes.

Reaction time and error rate decreased with practice. The speedup in reaction time was negatively accelerated, with the largest gains in the early trials, which is characteristic of a power function speedup (Logan, 1992b; Newell \& Rosenbloom, 1981). The learning curves were sharply inflected and shallow, which is typical of focused-attention conditions (see Logan, 1992a; Logan \& Etherton, 1994). Power functions were fitted to the mean reaction times. The predicted values appear as solid lines in Figure 1; the points represent the observed values. Measures of goodness of fit and the parameters of power functions fitted to the mean reaction times appear in Table 2. The fits were good; squared correlation $\left(r^{2}\right)$ between observed and predicted values was high, and the root mean squared deviation ( $m$ msd) between observed and predicted values was small.

Reaction times and error rates were subjected to 2 (target present vs. absent) $\times 16$ (practice block) analyses of variance (ANOVAs). Unless otherwise stated, in these and subsequent analyses $p<.05$. The reaction time ANOVA yielded significant main effects of target presence, $F(1,31)=4.60$, $M S E=5,473.85$, and practice block, $F(15,465)=14.79$, $M S E=2,759.85$, but no significant difference between them, $F(15,465)=1.25, M S E=1,193.68$. The error rate ANOVA yielded only a significant main effect of practice block, $F(15,465)=2.20, M S E=16.25$.

Recognition test. The main data of interest in the recognition test were the accuracies of the recognition judgments. The mean hit rates and correct rejection rates for target-present and target-absent displays appear in Table 3, as well as the corresponding reaction times. Recognition performance was not perfect but it was better than chance. The difference between hit rate and false-alarm rate was $22 \%$ for targetpresent displays and $27.0 \%$ for target-absent displays. The corresponding $d^{\prime}$ values were .801 and .828 for target-present and target-absent displays, respectively.

To compare recognition performance with chance, we performed one-way ANOVAs on hits minus false alarms and on recognition $d^{\prime}$ s, comparing target-present and target-absent displays. The error terms for these analyses were used to construct planned comparisons in which observed recognition performance was compared with chance (i.e., hits minus false alarms $=0.0 ; d^{\prime}=0.0$ ). Hit rate minus false-alarm rate was significantly greater than zero for target-present, $F(1,31)=$ $16.02, M S E=462.94$, and target-absent displays, $F(1,31)=$ $25.25, M S E=462.94, d^{\prime}$ was significantly greater than zero for target-present, $F(1,31)=19.31, M S E=0.53$, and targetabsent displays as well, $F(1,31)=20.65, M S E=0.53$.

\section{Discussion}

The training data suggested that some degree of automaticity was produced in the 16 training blocks because reaction time decreased as a power function of practice (Logan, 1988, 1992b). The memory test in transfer showed above-chance recognition of color-word pairs, which suggests that subjects learned about target color during training. Moreover, recognition performance was just as good for nontargets as it was for targets, which suggests that subjects learned color-word asso-

Table 2

Fits of Power Functions ( $\left.R T=a+b N^{-c}\right)$ to Mean Reaction Times From Experiments 1-8

\begin{tabular}{crrrrr}
\hline Experiment/target & \multicolumn{1}{c}{$r^{2}$} & $r m s d$ & $a$ & $b$ & $-c$ \\
\hline Exp. 1 & & & & & \\
Present & .956 & 4.52 & 637 & 90 & 1.116 \\
Absent & .913 & 8.18 & 647 & 111 & 1.356 \\
Exp. 2 & & & & & \\
Present & .972 & 10.08 & 739 & 256 & 0.981 \\
Absent & .960 & 7.98 & 671 & 164 & 1.404 \\
Exp. 3 & & & & & \\
Present & .934 & 6.31 & 623 & 99 & 1.608 \\
Absent & .901 & 10.47 & 635 & 132 & 1.478 \\
Exp. 4 & & & & & \\
Present & .982 & 6.88 & 666 & 260 & 0.514 \\
Absent & .973 & 8.87 & 630 & 224 & 1.269 \\
Exp. 5 & & & & & \\
Present & .915 & 6.95 & 627 & 94 & 8.091 \\
Absent & .973 & 4.50 & 646 & 113 & 2.213 \\
Exp. 6 & & & & & \\
Present & .978 & 10.89 & 629 & 369 & 0.506 \\
Absent & .965 & 10.69 & 625 & 235 & 1.666 \\
Exp. 7 & & & & & \\
Present & .970 & 9.90 & 678 & 257 & 0.697 \\
Absent & .954 & 9.65 & 631 & 183 & 1.458 \\
Exp. 8 & & & & & \\
Present & .977 & 9.51 & 731 & 277 & 0.825 \\
Absent & .947 & 9.11 & 665 & 161 & 2.187 \\
\hline
\end{tabular}

Note. $r^{2}=$ squared correlation between observed and predicted values; $\boldsymbol{m s s} \mathbf{d}=$ root mean squared deviation bewteen observed and predicted values; $a, b$, and $-c$ are parameters of the power function $\mathrm{RT}=a+b N^{-c}$, where $\mathrm{RT}=$ reaction time and $N=$ the number of practice trials. Exp. $=$ experiment. 
Table 3

Mean Reaction Times (in Milliseconds) and Accuracy

(Percentage of Hits and Percentage of Correct Rejections) for the Recognition Task in Experiments 1 and 2

\begin{tabular}{|c|c|c|c|c|c|}
\hline \multirow[b]{2}{*}{ Experiment/target } & \multirow{2}{*}{$\begin{array}{c}\text { Report in } \\
\text { training }\end{array}$} & \multicolumn{2}{|c|}{ Color same } & \multicolumn{2}{|c|}{ Color different } \\
\hline & & $M$ & $\mathbf{H ( \% )}$ & $M$ & CR (\%) \\
\hline $\begin{array}{l}\text { Exp. } 1 \\
\text { Present } \\
\text { Absent }\end{array}$ & No & $\begin{array}{l}1,284 \\
1,410\end{array}$ & $\begin{array}{l}75 \\
70\end{array}$ & $\begin{array}{l}1,500 \\
1,498\end{array}$ & $\begin{array}{l}46 \\
56\end{array}$ \\
\hline $\begin{array}{c}\text { Exp. } 2 \\
\text { Present } \\
\text { Absent }\end{array}$ & 'Yes & $\begin{array}{l}1,269 \\
1,400\end{array}$ & $\begin{array}{l}84 \\
72\end{array}$ & $\begin{array}{l}1,439 \\
1,549\end{array}$ & $\begin{array}{l}59 \\
50\end{array}$ \\
\hline
\end{tabular}

Note. $\quad$ Exp. $=$ experiment; $\mathrm{H}=$ hit; $\mathrm{CR}=$ correct rejection.

ciations independent of the words' membership in the target category.

Performance on the recognition test was above chance but it was not exceptional, considering that each color-word pair was presented 16 times during training. The difference between hit and false-alarm rates was $24.3 \%$, averaged across targetpresent and target-absent responses, which is about one quarter of the maximum difference that could occur if recognition were perfect. Recognition of color-word pairs may have been poor because subjects may have coded targets and nontargets as colored rather than as red or green (i.e., as attributes rather than as values). The attribute colored distinguishes targets and nontargets from distractors, which could be coded as uncolored (i.e., lacking the attribute color). The values red and green encode color more specifically, but that specificity may not have been necessary to focus attention on potential targets.

Alternatively, recognition of color-word pairs may have been poor because color may not have been a very effective retrieval cue. There were 16 targets in the target category but only two colors. Each color would be associated with 8 different targets. If all of the targets associated with a given color were retrieved, they may have impaired recognition more than they facilitated it. The different targets may have interfered with each other, making it harder to resolve which one was actually presented.

Nevertheless, recognition of color-word pairs was above chance, and that suggests that color information was encoded in the memory trace even when it was not reported explicitly. These results are consistent with the attention hypothesis, which predicts that color information should have been encoded because people had to pay attention to it.

\section{Experiment 2: Report in Training and Recognition Test at Transfer}

Experiment 2 was a replication of Experiment 1 in which subjects were required to report target color explicitly during training before transfer to the recognition memory test. Its purpose was to determine whether the requirement to report color explicitly would make subjects more likely to encode target color in the memory trace. Unlike Experiment 1, this experiment required subjects to attend to color values (red and green) rather than to color as an attribute (colored vs. uncolored), so they should be more likely to associate specific colors with specific words.

\section{Method}

Subjects. The subjects were 32 volunteers from the introductory psychology subject pool who received course credit for participating. Each subject was screened for red-green color blindness with the Ishihara (1987) test.

Apparatus and stimuli. The apparatus and stimuli were the same ones used in Experiment 1. The only difference was that the $z, x, c$, comma, period, and slash keys on the bottom row of the standard QWERTY keyboard were used to register responses.

Procedure. The procedure was much the same as in Experiment 1. There were 512 training trials in which each display contained one item in red or green and one in white. Particular words (targets and nontargets) were colored consistently throughout practice. There was one 32-trial transfer block in which colors of half targets and nontargets were reversed, and the colors of the other half remained the same as in training.

In training, subjects were told to classify the colored word as a member or a nonmember of a specified target category. If the word was a member of the target category, they were to indicate its color by pressing one key if it was red and another key if it was green. If the word was not a member of the target category, they were to press a single key, regardless of the word's color. Assignment to response keys was counterbalanced across subjects. Half of the subjects pressed two keys on the right-hand side to register target present (. for red and / for green) and one key on the left-hand side (c) to register target absent. The other half pressed two keys on the left-hand side to register target present ( $x$ for red and $z$ for green) and one key on the right-hand side to register target absent (,). All subjects were told to rest the index and middle fingers of their target-present hand and the index finger of their target-absent hand on the relevant keys throughout the experiment.

In transfer, the recognition memory task was the same as in Experiment 1. Subjects were told to decide whether the colored word appeared in the same color as it did in training. They reported their decisions by pressing one of two keys. Those who pressed $z, x$, and, in training pressed . (for same color) and / (for opposite color) in recognition. Those who pressed period, slash, and $c$ in training pressed $x$ (for same color) and $z$ (for opposite color) in recognition.

\section{Results}

Training. The mean reaction times and percentage of error scores for target-present and target-absent responses in the 16 training blocks are presented in Figure 2. Reaction times were slower for target-present than for target-absent responses, reflecting the extra requirement to report color. Error rate was higher for target-present responses, suggesting that people had difficulty with the additional choice of color.

Reaction time and error rate decreased with practice. The speedup in reaction time followed a power function, characteristic of automaticity (Logan, 1992b; Newell \& Rosenbloom, 1981). Power functions were fitted to the mean reaction times. The predicted values appear as solid lines in Figure 2; the points represent the observed values. Measures of goodness of fit and the parameters of power functions fitted to the mean reaction times appear in Table 2. Again, the fits were good.

Reaction times and error rates were subjected to 2 (target present vs. absent) $\times 16$ (practice block) ANOVAs. The reaction time ANOVA found significant main effects of target 


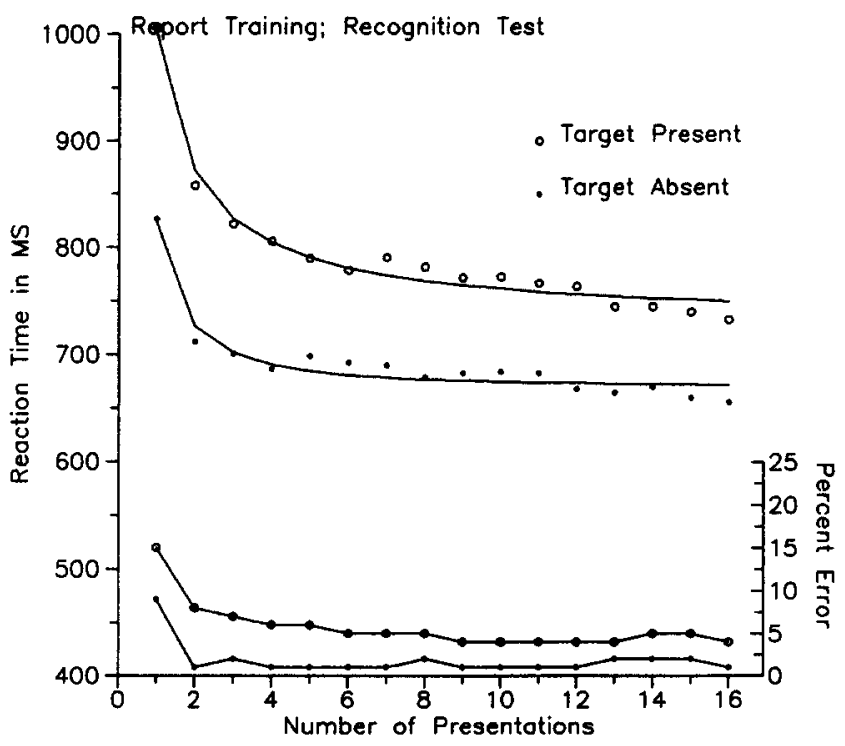

Figure 2. Mean reaction times (top two lines, left-hand $y$ axis) and error rates (bottom two lines, right-hand $y$ axis) for target-present (open circles) and target-absent (filled circles) responses from the training phase of Experiment 2 as a function of number of presentations (for the reaction times, the lines represent the best fitting power function, and the circles represent the observed data).

presence, $F(1,31)=59.23, M S E=42,579.89$, and practice block, $F(15,465)=22.18, M S E=7,721.70$, and a significant interaction between target presence and practice, $F(15,465)=$ 3.93, $M S E=2,140.54$. The error rate ANOVA found significant main effects of target presence, $F(1,31)=26.96, M S E=$ 199.33 , and practice block, $F(15,465)=14.57, M S E=24.95$. The interaction between target presence and practice was not significant, $F(15,465)=1.44, M S E=31.24$.

Recognition test. As before, the main data of interest in the recognition test were the accuracies of the recognition judgments. The mean hit rates and correct rejection rates for target-present and target-absent displays appear in Table 3, along with the corresponding reaction times. Recognition performance was better than chance and better for targetpresent displays (hit rate minus false-alarm rate $=42 \%$; $d^{\prime}=1.63$ ) than for target-absent displays (hits minus falsealarm rate $=23 \% ; d^{\prime}=0.82$ ).

The data were analyzed in two one-way ANOVAs: one on hits minus false alarms and one on recognition $d$ 's, comparing target-present and target-absent displays. The error terms for these analyses were used to construct planned comparisons in which observed recognition performance was compared with chance. Hit rate minus false-alarm rate was significantly greater than zero for target-present, $F(1,31)=39.40, M S E=$ 711.03 , and target-absent displays, $F(1,31)=12.20, M S E=$ 711.03. $d$ ' was significantly greater than zero for target-present, $F(1,31)=61.92, M S E=0.68$, and target-absent displays as well, $F(1,31)=15.84, M S E=0.68$.

The data were compared with the recognition data from Experiment 1 in two 2 (target present vs. absent) $\times 2$ (Experiment 1 vs. Experiment 2) ANOVAs: one on hits minus false alarms and one on $d^{\prime}$ s. The main effect of experiments was not significant in the analysis of hits minus false alarms, $F(1,62)=2.73, M S E=803.66$, but it was in the analysis of $d^{\prime}$, $F(1,62)=4.00, M S E=1.34$. The interaction between experiments and target presence was significant in both analyses: $F(1,62)=7.89, M S E=586.98$ for hits minus false alarms and $F(1,62)=9.09, M S E=0.61$ for $d^{\prime}$ 's. The main difference between experiments was with target-present displays, in which subjects in Experiment 2 had to report color in training, but subjects in Experiment 1 did not. Target-absent displays did not require color report in either experiment and showed no difference between experiments.

\section{Discussion}

The training data showed evidence of automatization, in that reaction time decreased as a power function of practice (Logan, 1988, 1992b). The transfer data showed that colorword associations were encoded into memory during automatization because recognition performance was well above chance. Memory performance was much better for target-present items, which required explicit color report in training, than for target-absent responses in this experiment and both targetpresent and target-absent items in Experiment 1, which did not require explicit color report. This confirms the prediction derived from the attention hypothesis and motivates the use of the requirement to report color explicitly to guarantee encoding of color in the subsequent automaticity experiments.

Recognition of color-word pairs was better in Experiment 2 than in Experiment 1, but it was still nowhere near ceiling. That is surprising because each color-word pair was presented 16 times during training. The requirement to report colors explicitly in Experiment 2 rules out the possibility that subjects encoded the attribute colored rather than specific color values red and green, as they may have in Experiment 1. Nevertheless, the possibility remains that memory performance was poor because there were 16 words associated with each color, so color may not have been a very unique retrieval cue (cf. the Discussion section of Experiment 1).

\section{Experiment 3: No Report at Training or Transfer}

Experiments 1 and 2 demonstrated that the focusedattention orienting task is sufficient to cause color information to be encoded into memory during automatization. The next set of experiments was designed to determine whether color information would be expressed in automatic performance at retrieval time. Experiments 3-6 were part of a $2 \times 2$ factorial design that crossed the requirement to report color at training with the requirement to report color at transfer. Experiment 3 was first in the series.

Subjects performed the focused-attention category search task for 16 blocks in which colors and words were paired consistently. Then they transfered to a 32-trial transfer block in which the colors were reversed (e.g., if Steel appeared in red in training, it appeared in green in transfer). If the retrieval task at transfer-automatic categorical decision-was sensitive to the presence of color in the memory trace, then performance should be disrupted by the change in color. If the retrieval task was not sensitive to color in the memory trace, performance should not be disrupted. 


\section{Method}

Subjects. Thirty-two volunteers from an introductory psychology course participated in the experiment for course credit. None had served in the earlier experiments, and each was screened for red-green color blindness with the Ishihara (1987) test.

Apparatus and stimuli. The apparatus and stimuli were the same as in the previous experiments. The were three main differences: First, different keys were pressed to report target presence and absence ( $z$ and /). Second, the word pair was presented for $1,000 \mathrm{~ms}$ throughout the experiment, in both training and transfer. Third, the composition of color-word pairs in the transfer block was different; each target and nontarget appeared in the color opposite to the one it appeared in during training (e.g., if Steel appeared in red in training, it appeared in green at transfer).

Procedure. Training consisted of 16 blocks of 32 trials with consistent pairings of colors and words, just like in the previous experiments. After the training blocks, there was one 32-trial transfer block in which each target and nontarget was presented once in the color opposite to the one it appeared in during training. Half of the subjects were told to indicate target presence with their right hand and target absence with their left hand, and half were told the opposite. All subjects were told to rest their index finger of their left and right hands lightly on the keys throughout the experiment.

\section{Results}

Training. Mean reaction times and error rates for targetpresent and target-absent responses are presented as a function of training block in Figure 3. Reaction times were faster for target-present than for target-absent responses, and error rate was higher for target-present responses.

Reaction time and error rate decreased with practice. The speedup in reaction time followed a power function characteristic of automaticity (Logan, 1988, 1992b). Power functions were fitted to the mean reaction times. The predicted values appear as solid lines in Figure 3; the points represent the observed values. Measures of goodness of fit and the parameters of power functions fitted to the mean reaction times appear in Table 2. The fits were good, as in the previous experiments.

Reaction times and error rates were subjected to 2 (target present vs. absent) $\times 16$ (practice block) ANOVAs, in which $p<.05$ was used as the significance level. The ANOVA on the reaction times found significant main effects of target presence, $F(1,31)=15.36, M S E=5,495.53$, and practice block,

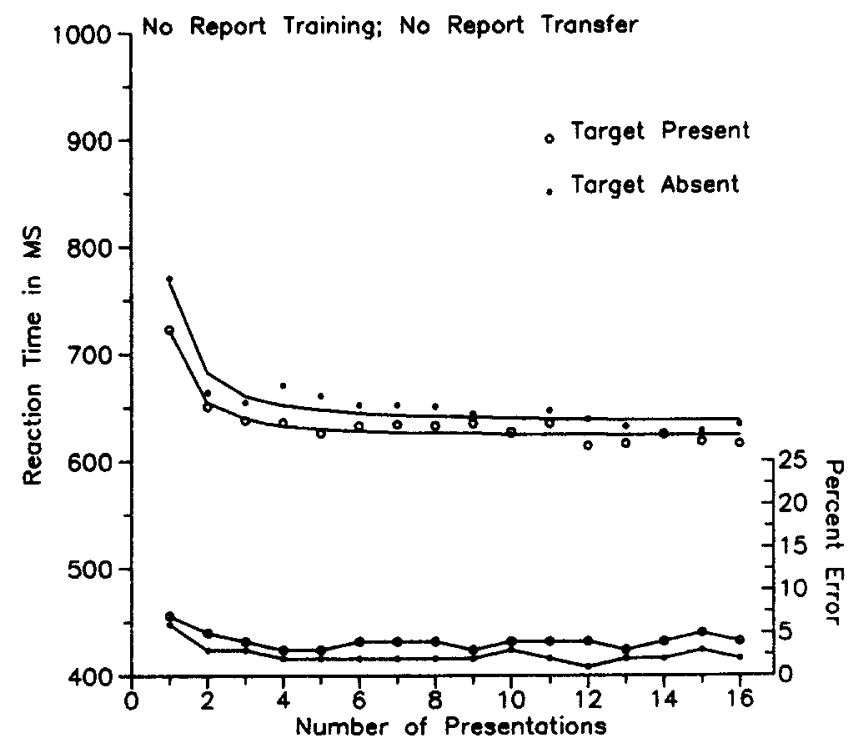

Figure 3. Mean reaction times (top two lines, left-hand $y$ axis) and error rates (bottom two lines, right-hand $y$ axis) for target-present (open circles) and target-absent (filled circles) responses from the training phase of Experiment 3 as a function of number of presentations (for the reaction times, the lines represent the best fitting power function, and the circles represent the observed data).

$F(15,465)=24.26, M S E=2,301.57$. The interaction between target presence and practice was significant as well, $F(15,465)=$ $3.38, M S E=781.74$. The ANOVA on the error rates found significant main effects of target presence, $F(1,31)=5.12$, $M S E=91.52$, and practice block, $F(15,465)=4.89, M S E=$ 16.33. The interaction between target presence and practice was not significant, $F(15,465)=1.02, M S E=16.70$.

Transfer. Mean reaction times and error rates for the last training block and the transfer block are presented in Table 4 for target-present and target-absent responses. Subjects did not report target color in training or in transfer, and the cost of changing color at transfer was negligible.

The mean reaction times and error rates were analyzed in 2 (color same vs. different) $\times 2$ (target present vs. absent) ANOVAs. The small cost in reaction time was not significant

Table 4

Costs From Transfer for Mean Reaction Times in Milliseconds and Percentage of Error (in Parentheses) in Experiments 3-6

\begin{tabular}{|c|c|c|c|c|c|c|}
\hline \multirow[b]{2}{*}{ Experiment } & \multicolumn{2}{|c|}{ Report in } & \multicolumn{2}{|c|}{ Color } & \multirow[b]{2}{*}{ Cost } & \multirow[b]{2}{*}{ Target } \\
\hline & Training & Transfer & Same & Different & & \\
\hline Exp. 3 & No & No & $\begin{array}{l}615(3) \\
634(2)\end{array}$ & $\begin{array}{l}619(3) \\
628(2)\end{array}$ & $\begin{array}{r}4(0) \\
-6(0)\end{array}$ & $\begin{array}{l}\text { Present } \\
\text { Absent }\end{array}$ \\
\hline Exp. 4 & Yes & Yes & $\begin{array}{l}727(6) \\
629(2)\end{array}$ & $\begin{array}{l}810(11) \\
642(2)\end{array}$ & $\begin{array}{l}83(5) \\
13(0)\end{array}$ & $\begin{array}{l}\text { Present } \\
\text { Absent }\end{array}$ \\
\hline Exp. 5 & No & Yes & $\begin{array}{l}784(14) \\
659(4)\end{array}$ & $\begin{array}{l}782(11) \\
677(3)\end{array}$ & $\begin{array}{r}-2(-3) \\
18(-1)\end{array}$ & $\begin{array}{l}\text { Present } \\
\text { Absent }\end{array}$ \\
\hline Exp. 6 & Yes & No & $\begin{array}{l}609(5) \\
605(3)\end{array}$ & $\begin{array}{l}609(4) \\
604(1)\end{array}$ & $\begin{array}{c}0(1) \\
-1(-2)\end{array}$ & $\begin{array}{l}\text { Present } \\
\text { Absent }\end{array}$ \\
\hline
\end{tabular}

Note. $\quad$ Cost $=$ different - same. Exp. $=$ experiment. 
statistically. There were no significant effects in either the reaction time or the error rate ANOVA.

\section{Discussion}

The training data suggested that automaticity was produced because reaction time decreased as a power function of practice (Logan, 1988, 1992b). The transfer data suggested that the memory traces that supported automatic performance were not sensitive to the colors that guided attention to the appropriate word. Apparently, the retrieval task, which did not require explicit report during transfer, was not sensitive to the presence of color information in the memory trace.

\section{Experiment 4: Report in Training and Transfer}

Experiment 4 was a replication of Experiment 3, except that subjects were required to report the color of the words that matched the target category in both training and transfer. The attention hypothesis predicts that an encoding task that requires explicit report of color information should cause color information to be encoded in the memory trace, and a retrieval task that also requires explicit report of color information should be sensitive to the presence of color information in the memory trace. Thus, performance should be disrupted when the colors change at transfer.

\section{Method}

Subjects. The subjects were 32 volunteers from the same introductory psychology subject pool used in the previous experiments. None had served in the previous experiments, and all were screened for red-green color blindness with the Ishihara (1987) test.

Apparatus and stimuli. The apparatus and stimuli were the same ones used in the previous experiments. The only difference was that the $z, x$, period, and slash keys on the bottom row of the standard QWERTY keyboard were used to register responses.

Procedure. The procedure was the same as in Experiment 3. There were 512 training trials in which each display contained one item in red or green and one in white. Particular words (targets and nontargets) were colored consistently throughout practice. There was one 32-trial transfer block in which colors of targets and nontargets were reversed. Words that appeared in red in training now appeared in green; words that appeared in green now appeared in red.

Assignment to response keys was counterbalanced across subjects. Half of the subjects pressed two keys on the right-hand side to register target present (. for red and / for green) and one key on the left-hand side $(z)$ to register target absent. The other half pressed two keys on the left-hand side to register target present ( $x$ for red and $z$ for green) and one key on the right-hand side to register target absent (/). All subjects were told to rest the index and middle fingers of their target-present hand and the index finger of their target-absent hand on the relevant keys throughout the experiment. Assignment of responses to keys was constant throughout training and transfer.

\section{Results}

Training. The mean reaction times and percentage of error scores for target-present and target-absent responses in the 16 training blocks are presented in Figure 4. Reaction times were slower for target-present than for target-absent responses, reflecting the extra requirement to report color. Error rate was

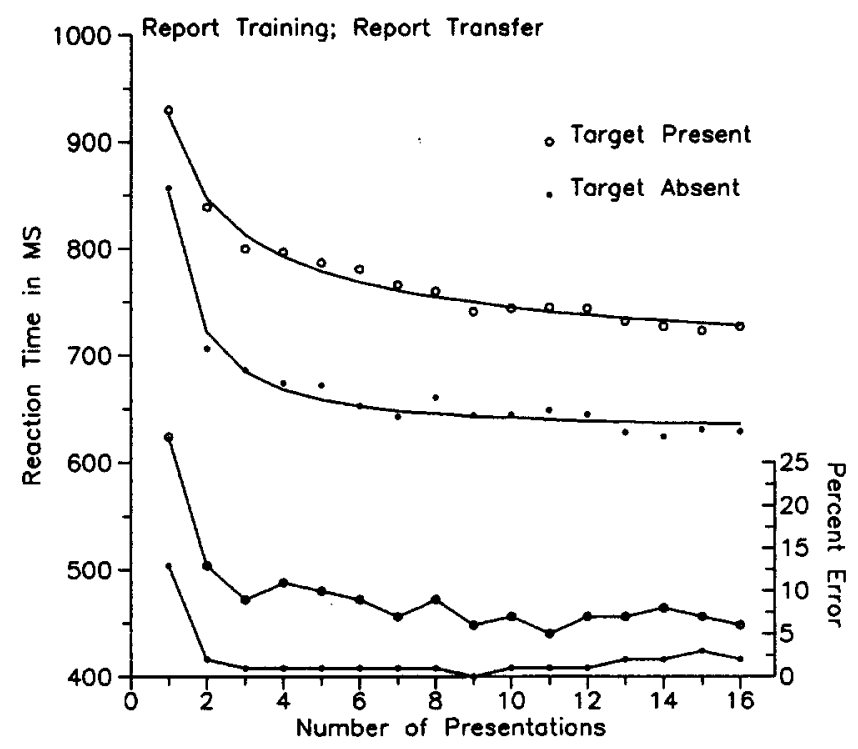

Figure 4. Mean reaction times (top two lines, left-hand $y$ axis) and error rates (bottom two lines, right-hand $y$ axis) for target-present (open circles) and target-absent (filled circles) responses from the training phase of Experiment 4 as a function of number of presentations (for the reaction times, the lines represent the best fitting power function, and the circles represent the observed data).

higher for target-present responses, suggesting that people had difficulty with the additional choice of color.

Reaction time and error rate decreased with practice. The speedup in reaction time followed a power function characteristic of automaticity (Logan, 1992b; Newell \& Rosenbloom, 1981). Power functions were fitted to the mean reaction times. The predicted values appear as solid lines in Figure 4; the points represent the observed values. Measures of goodness of fit and the parameters of power functions fitted to the mean reaction times appear in Table 2. Again, the fits were good.

Reaction times and error rates were subjected to 2 (target present vs. absent) $\times 16$ (practice block) ANOVAs. The reaction time ANOVA found significant main effects of target presence, $F(1,31)=75.79, M S E=37,979.46$, and practice block, $F(15,465)=44.63, M S E=4,192.29$, and a significant interaction between target presence and practice, $F(15,465)=$ $1.78, M S E=1,418.59$. The error rate ANOVA found significant main effects of target presence, $F(1,31)=38.46, M S E=$ 329.47 , and practice block, $F(15,465)=22.40, M S E=46.57$. The interaction between target presence and practice was significant, $F(15,465)=4.07, M S E=31.45$.

Transfer. The mean reaction times and error rates for the last training block and the transfer block are presented in Table 4. Subjects reported target color in training and transfer, and there was a large cost for target-present trials when color changed at transfer. The cost for target-absent trials, which did not require color report, was negligible.

The mean reaction times and error rates were analyzed in 2 (color same vs. different) $\times 2$ (target present vs. absent) ANOVAs. In the reaction time ANOVA, there was a significant main effect of color change, $F(1,31)=21.83, M S E=$ $3,410.06$, and target presence, $F(1,31)=69.97, M S E=$ 
$8,080.05$, and a significant interaction between them, $F(1,31)=$ $11.75, M S E=3,350.34$. The error rate ANOVA showed similar effects: a significant main effect of color change, $F(1$, $31)=5.29, M S E=26.52$, and target presence, $F(1,31)=$ $33.92, M S E=37.59$, and a significant interaction between them, $F(1,31)=6.05, M S E=26.79$.

\section{Discussion}

The training data showed evidence of automatization in that reaction time sped up following a power function (Logan, 1988, 1992b). The target-present data at transfer suggested that the memory traces that supported automatic performance contained color information. Apparently, the requirement to report color caused color to be encoded into the memory trace during the acquisition of automaticity and retrieved from it during the expression of automaticity in performance. The target-absent data were interesting because they showed no cost of changing color at transfer. They replicated Experiment 3 , suggesting that color was not retrieved when it did not have to be reported.

\section{Experiment 5: No Report in Training and Report at Transfer}

In Experiment 5 we paired the training condition of Experiments 1 and 3 with the transfer condition of Experiment 4; subjects reported target color explicitly in transfer but not in training. The purpose was to determine whether an encoding task that did not require explicit report of color would nevertheless cause color information to be encoded in the memory trace. If it did, transfer performance should be disrupted when target color changed, as in Experiment 4. If the encoding task did not cause color to be encoded, then transfer performance would be unaffected by color change, as in Experiment 3.

\section{Method}

Subjects. The subjects were 32 volunteers from the introductory psychology subject pool used in the previous experiments. None had served in a previous experiment, and each one was screened for red-green color blindness with the Ishihara (1987) test.

Apparatus and stimuli. The apparatus and stimuli were the same as those used in the previous experiments. The training trials used the same response keys as Experiment 3 ( $z$ and /), and the transfer trials used the same response keys as Experiment $4(z, x$, period, and slash).

Procedure. The procedure during training was the same as the training procedure in Experiments 1 and 3: Subjects pressed single keys to report target presence or absence. At transfer, subjects pressed one of two keys to report the color of the target if a target was present or they pressed a single key regardless of color if no target was present. Mapping was consistent from training to transfer in that the same hands were used to indicate target presence and absence. Thus, people who pressed / for target presence with their right hand in training would press . for red targets and / for green targets with their right hand in transfer.

The transfer block was different from the one used in the previous experiments. It involved 64 trials, 32 with targets and nontargets colored in the same way they were colored in training (i.e., words colored red in training remained red, and words colored green remained green) and 32 with targets and nontargets colored oppositely (i.e., words colored red in training appeared in green, and words colored green appeared in red). This procedure was necessary because the response requirements changed from training to transfer, so it was no longer possible to assess the cost of changing color by comparing the last training block to the transfer block. The cost of changing color was assessed by comparing same-color with different-color trials within the transfer block, under identical response requirements.

\section{Results}

Training. Mean reaction times and error rates for targetpresent and target-absent responses are plotted as a function of practice block in Figure 5. Reaction times were faster for target-present responses and error rates were higher, replicating Experiments 1 and 3. Reaction time and error rate decreased with practice. Most of the reduction in reaction time occurred between the first and the second block; reaction time was relatively stable after that. Nevertheless, the data were well fit by power functions. The solid lines in Figure 5 represent the fitted functions; the points represent the observed data. Measures of goodness of fit and the parameters of the fitted functions appear in Table 2.

Reaction times and error rates were subjected to 2 (target present vs. absent) $\times 16$ (practice block) ANOVAs. The ANOVA on the reaction times found significant main effects of target presence, $F(1,31)=26.65, M S E=5,544.00$, and practice block, $F(15,465)=9.50, M S E=4,613.58$, and a significant interaction between target presence and practice, $F(15,465)=1.69, M S E=1,194.08$. The ANOVA on the error rates found significant main effects of target presence, $F(1,31)=5.18, M S E=147.28$, and practice block, $F(15,465)=$

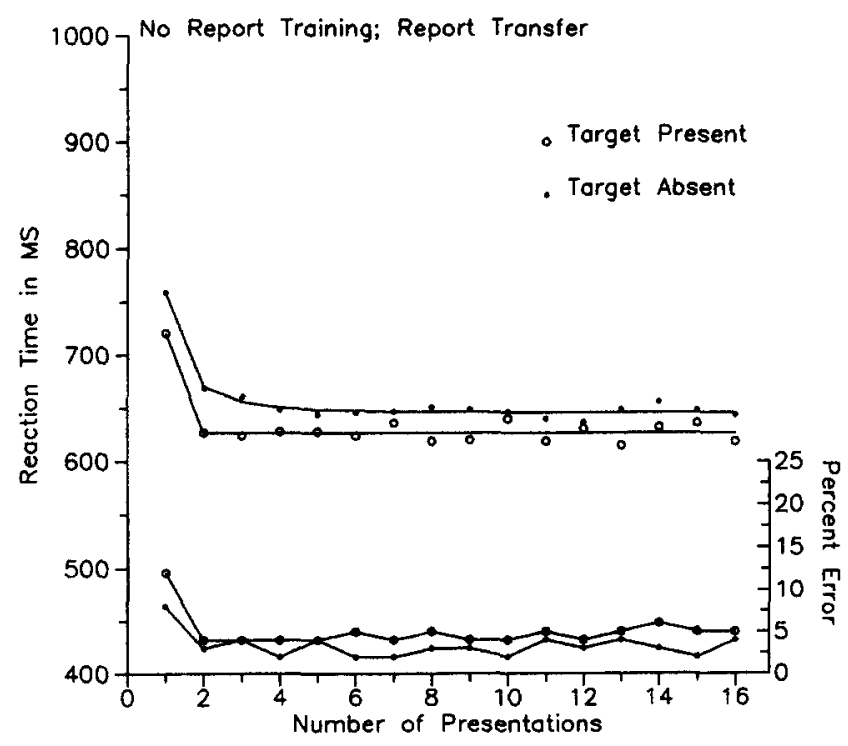

Figure 5. Mean reaction times (top two lines, left-hand $y$ axis) and error rates (bottom two lines, right-hand $y$ axis) for target-present (open circles) and target-absent (filled circles) responses from the training phase of Experiment 5 as a function of number of presentations (for the reaction times, the lines represent the best fitting power function, and the circles represent the observed data). 
$6.57, M S E=24.17$, but no significant interaction between them $(F<1.0)$.

Transfer. Mean reaction times and error rates for sameand different-color trials in the transfer block are presented in Table 4. Subjects in this experiment did not report target color in training, but they did at transfer. Nevertheless, cost for changing color was negligible for both target-present and target-absent displays. The only significant effects in 2 (color same vs. different) $\times 2$ (target present vs. absent) ANOVAs on mean reaction times and error rates were for target presence: for reaction time, $F(1,31)=70.17, M S E=6,019.30$; for accuracy, $F(1,31)=9.56, M S E=273.93$.

\section{Discussion}

Automaticity developed with training, as in the previous experiments, evidenced by a power function speedup in reaction time (Logan, 1988, 1992b). The transfer data suggested that color information was not encoded into the memory trace when the training task did not require it to be reported explicitly. The data from Experiment 4 suggested that the retrieval task at transfer, which required explicit report of target color, would have been sensitive to the presence of color information in the memory trace if it had been encoded. The fact that color change had no effect on performance suggests that color information was not encoded in the memory trace.

\section{Experiment 6: Report in Training and No Report at Transfer}

Experiment 6 replicated the training conditions of Experiments 2 and 4 and the transfer conditions of Experiment 3; subjects reported target color explicitly in training but not at transfer. The purpose was to see whether the retrieval task at transfer was sensitive to the presence of color information in the memory trace. If it was, transfer performance should be disrupted when target color changed, as it was in Experiment 4. If the retrieval task was not sensitive to color in the memory trace, then transfer performance should be unaffected by color change, as it was in Experiment 3.

\section{Method}

Subjects. The subjects were 32 volunteers from the introductory psychology subject pool sampled in the previous experiments. None had served in the previous experiments, and each one was screened for red-green color blindness with the Ishihara (1987) test.

Apparatus and stimuli. The apparatus and stimuli were the same as those used in Experiments 3 and 4. During training, subjects responded using the $z, x$, period, and slash keys, as in Experiment 4. At transfer, they responded using the $z$ and slash keys, as in Experiment 3.

Procedure. The procedure during training was the same as the training procedure in Experiments 2 and 4: Subjects reported the color of targets if targets were present and made a single response regardless of color if no target was present. At transfer, they pressed a single key ( / or $z$ ) to indicate target presence and another single key $(z$ or $/$ ) to indicate target absence. Mapping was consistent from training to transfer in that the same hands were used to indicate target presence and absence. Thus, subjects who pressed . for red targets and / for green targets with their right hand would press / for target presence with their right hand.
The transfer block was like the one in Experiment 5. It involved 64 trials, 32 with targets and nontargets colored in the same way they were colored in training and 32 with targets and nontargets colored oppositely. This procedure allowed us to assess the cost of changing color under the same response requirements (i.e., when color did not have to be reported).

\section{Results}

Training. The mean reaction times and error rates are presented in Figure 6. The data resembled those of Experiments 2 and 4: Reaction time was longer for target-present than for target-absent responses, reflecting the extra degree of choice involved, and error rates were higher for target-present responses as well. Again, both reaction time and error rate decreased with practice. The decrease in reaction time followed a power function. The solid lines in Figure 6 represent the fitted power function; the points represent the observed data. Measures of goodness of fit and the parameters of the fitted functions are presented in Table 2. Again, the fits were good.

Reaction times and error rates were subjected to 2 (target present vs. absent) $\times 16$ (practice block) ANOVAs. The ANOVA on the reaction times found significant main effects of target presence, $F(1,31)=145.05, M S E=28,833.02$, and practice block, $F(15,465)=58.49, M S E=4,805.81$, and a significant interaction between them, $F(15,465)=6.10$, $M S E=2,324.75$. The ANOVA on the error rates found similar results: significant main effects of target presence, $F(1,31)=$ $42.16, M S E=232.35$, and practice block, $F(15,465)=14.31$, $M S E=33.98$, and a significant interaction between them, $F(15,465)=2.00, M S E=24.71$.

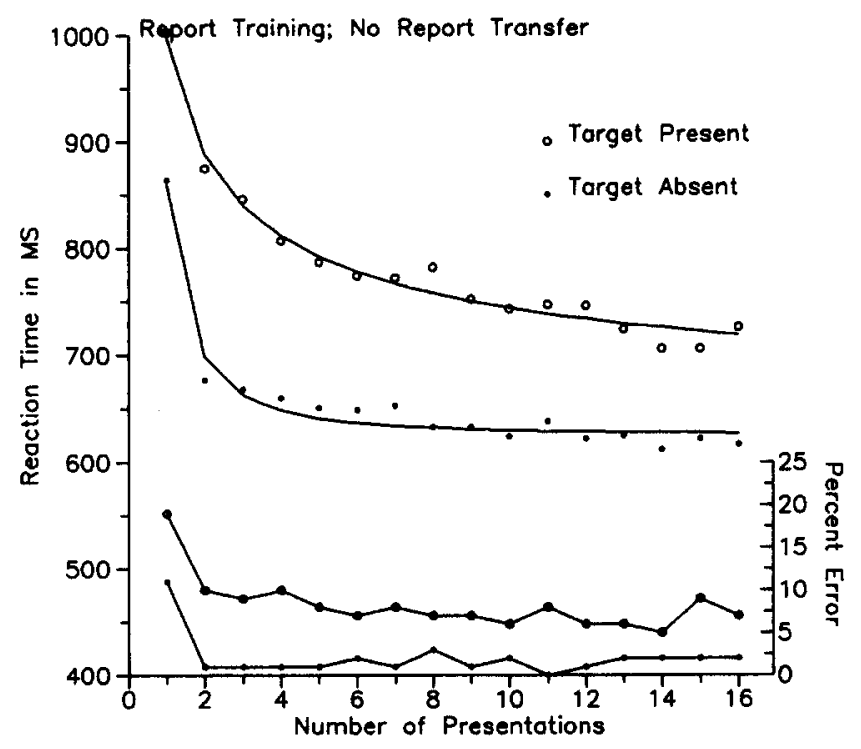

Figure 6. Mean reaction times (top two lines, left-hand $y$ axis) and error rates (bottom two lines, right-hand $y$ axis) for target-present (open circles) and target-absent (filled circles) responses from the training phase of Experiment 6 as a function of number of presentations (for the reaction times, the lines represent the best fitting power function, and the circles represent the observed data). 
Transfer. Mean reaction times and error rates for sameand different-color trials in the transfer block are presented in Table 4. Subjects in this experiment reported target color at training but not in transfer. They showed a negligible cost of changing color at transfer in both target-present and targetabsent trials. The reaction times and error rates were analyzed in 2 (color same vs. different) $\times 2$ (target present vs. absent) ANOVAs. There were no significant effects in either ANOVA.

\section{Discussion}

The training data suggested that automaticity developed with practice because reaction time decreased as a power function of the number of presentations. The transfer data suggest that a retrieval task that does not require report of color is not sensitive to color information in the memory trace. The data from Experiment 4 suggest that the requirement to report color during training was likely to have caused color to be encoded in the memory trace. The fact that changing color did not affect the expression of automaticity in the transfer block of the present experiment suggests that the retrieval task was not sensitive to color information in the trace.

\section{Experiment 7: Report in Training and Report With Changed Responses at Transfer}

Experiments 3-6 suggested that color was important in the memory trace only if the encoding task and the retrieval task both required report of color (i.e., the procedure of Experiment 4). There are three alternative interpretations of those results: Explicit color reports could lead to associations between words and colors, between words and responses, or between words, responses, and colors. All three of these associations were "broken" by switching to opposite colors in the transfer block of Experiment 4. Experiments 7 and 8 were designed to discriminate between the alternatives.

Experiment 7 required report of color in training and in transfer, but the response keys used for report changed between training and transfer. Subjects pressed a different set of keys in transfer. Half of the transfer words appeared in the same color as in training, and half appeared in the opposite color. The first hypothesis, that explicit report in training produces associations between words and colors, predicts a cost from changing color but no cost from changing responses. Target-present same-color responses should be just as fast in transfer as target-present responses were in the last training block. Target-present opposite-color responses should be slower in transfer than in the last training block.

The second hypothesis, that explicit report in training produces associations between words and responses, predicts a cost of changing response keys at transfer but no cost of changing color. Target-present responses should be slower in transfer than in the last training block because people press new keys and associations between words, and the old keys will no longer be beneficial. Moreover, there should be no difference between same- and opposite-color target-present responses in transfer because color is not part of the memory trace.
The third hypothesis, that explicit report in training produces associations between words, colors, and responses, makes the same predictions as the second hypothesis. Targetpresent responses should be slower in transfer than in training, and same- and opposite-color responses should not differ in transfer because the combinations of colors, words, and responses that were acquired in training do not appear in transfer. Thus, Experiment 7 distinguishes the first hypothesis from the second and third. Experiment 8 will distinguish the second from the third, if need be.

\section{Method}

Subjects. The subjects were 32 volunteers from the introductory psychology subject pool who were screened for red-green color blindness with the Ishihara (1987) test. None had served in Experiments 1-6.

Apparatus and stimuli. The apparatus and stimuli were the same as those used in the previous experiments. The response keys were slightly different, however. This experiment used $z, x, c$, comma, period, and slash keys, which were the three leftmost and three rightmost keys on the bottom row of the standard QWERTY keyboard.

Procedure. The procedure was the same as in Experiment 4, in that target color had to be reported in training and in transfer, but it was different in that there were 64 transfer trials, including both samecolor and different-color word pairs (as in Experiments 5 and 6). In addition, the physical responses required to report target color and target absence were different in transfer than in training. Half of the subjects pressed the . key for red targets, the / key for green targets, and the $c$ key for nontargets during training. The other half pressed the $x$ key for red targets, the $z$ key for green targets, and the, key for nontargets during training. At transfer, each group changed mapping, adopting the one that the other group was trained on. Notice that for both groups, the keys used in transfer were different from the ones used in training.

\section{Results}

Training. The mean reaction times and percentage of error scores for target-present and target-absent responses in the 16 training blocks are presented in Figure 7. As in Experiments 2, 4 , and 6, reaction times were slower for target-present than for target-absent responses and error rate was higher, reflecting the difficulty that subjects had with the additional choice of color.

Reaction time and error rate decreased with practice. The speedup in reaction time followed a power function, as in the previous experiments. Power functions were fitted to the mean reaction times; the predicted values appear as solid lines in Figure 7. The points represent the observed values. Measures of goodness of fit and the parameters of power functions fitted to the mean reaction times appear in Table 2. Again, the fits were good.

Reaction times and error rates were subjected to 2 (target present vs. absent) $\times 16$ (practice block) ANOVAs. The reaction time ANOVA found significant main effects of target presence, $F(1,31)=264.51, M S E=9,934.38$, and practice block, $F(15,465)=62.02, M S E=2,817.02$, and a significant interaction between target presence and practice, $F(15,465)=$ $3.32, M S E=1,512.24$. The error rate ANOVA found signifi- 


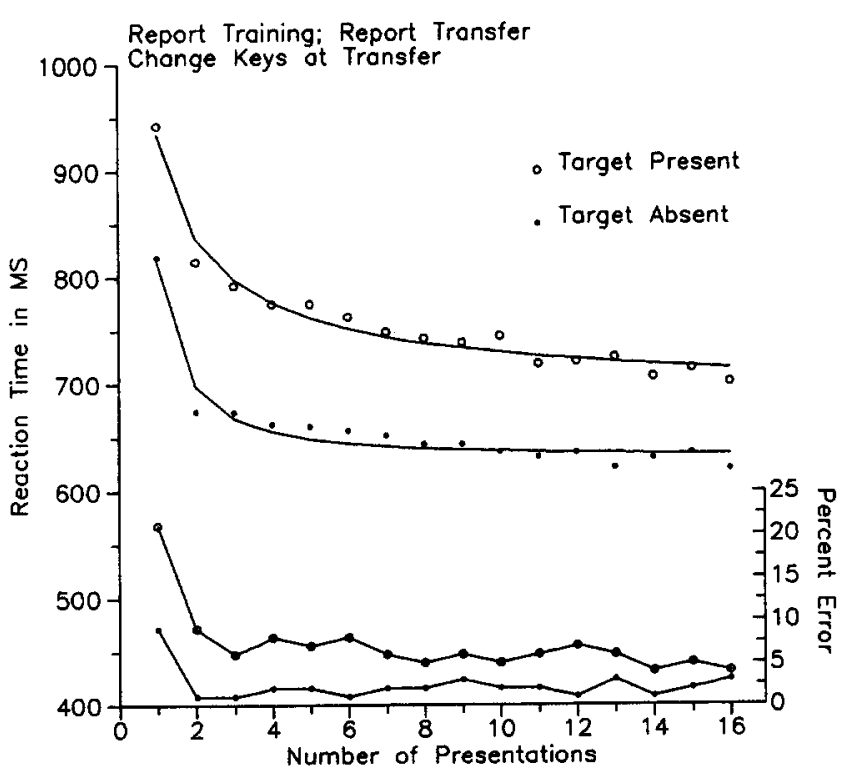

Figure 7. Mean reaction times (top two lines, left-hand $y$ axis) and error rates (bottom two lines, right-hand $y$ axis) for target-present (open circles) and target-absent (filled circles) responses from the training phase of Experiment 7 as a function of number of presentations (for the reaction times, the lines represent the best fitting power function, and the circles represent the observed data).

cant main effects of target presence, $F(1,31)=38.46, M S E=$ 329.47 , and practice block, $F(15,465)=22.40, M S E=46.57$. The interaction between target presence and practice was significant, $F(15,465)=4.07, M S E=31.45$.

Transfer. Table 5 contains the mean reaction times and error rates for the last training block and same-color and opposite-color trials in the transfer block. Subjects reported target color in training as well as in transfer, but the stimulusto-response mapping changed at transfer. There was an increase in reaction time from training to transfer for both target-present and target-absent trials, but there was no cost for changing color at transfer for either trial type.

The mean reaction times and error rates were analyzed in 3 (presentation: last training block vs. color same in transfer vs. color different in transfer) $\times 2$ (target present vs. absent) ANOVAs on the mean reaction times and error rates. In the reaction time ANOVA, there was a significant main effect of presentation, $F(2,62)=15.95, M S E=1,973.89$, and target presence, $F(1,31)=207.78, M S E=1,796.58$. Planned comparisons revealed slower reaction times in transfer than in the last training block for target-present responses, $F(1,62)=$ $30.05, M S E=1,437.72$, and target-absent responses, $F(1,62)=$ $15.67, M S E=1,437.72$. Further planned comparisons revealed no difference between same-color and opposite-color trials in the transfer block for target-present responses $(F<1.0)$ and for target-absent responses, $F(1,62)=1.35, M S E=1,437.72$.

In the error rate ANOVA, the main effect of target presence, $F(1,31)=25.53, M S E=25.71$, and the interaction between target presence and presentation were significant, $F(2,62)=6.62, M S E=19.82$. Planned comparisons showed lower accuracy in transfer than in the last training block for target-present responses, $F(1,62)=13.19, M S E=19.82$, but not for target-absent responses $(F<1.0)$. Further planned comparisons showed no effect of same- versus opposite-color trials in the transfer block for target-present or target-absent responses (both $F \mathrm{~s}<1.0$ ).

\section{Discussion}

The training data showed evidence of automatization in that reaction time sped up following a power function (Logan, $1988,1992 \mathrm{~b}$ ). The target-present data showed a cost between the last training block and the transfer block, in which responses changed. The transfer block itself showed no greater cost for opposite-color responses than for same-color responses. These results rule out the hypothesis that associations between colors and words were responsible for the transfer costs seen in Experiment 4. They are consistent with the hypothesis that associations between words and responses were responsible and with the hypothesis that associations between colors, words, and responses were responsible. Experiment 8 was designed to distinguish between the latter two hypotheses.

\section{Experiment 8: Report in Training and Reverse Response Mapping in Transfer}

In Experiment 8 we required report of target color in training and in transfer but at transfer, the keys used for report were reversed. Subjects who pressed / for red and . for green in

Table 5

Mean Reaction Times in Milliseconds and Percentage of Error (in Parentheses) for the Last

Training Session and for Color-Same and Color-Different Trials in the Transfer Block of Experiments 7 and 8

\begin{tabular}{cccccc}
\hline Experiment/target & $\begin{array}{c}\text { Last training } \\
\text { block }\end{array}$ & $\begin{array}{c}\text { Transfer } \\
\text { same }\end{array}$ & $\begin{array}{c}\text { Transfer } \\
\text { different }\end{array}$ & Cost $_{1}$ & Cost $_{2}$ \\
\hline Exp. 7 & $702(4)$ & $744(7)$ & $748(9)$ & $44(4)$ & $4(2)$ \\
Present & $621(3)$ & $659(3)$ & $648(2)$ & $33(0)$ & $-11(-1)$ \\
Absent & & & & & \\
Exp. 8 & $754(5)$ & $820(8)$ & $805(10)$ & $59(4)$ & $-15(2)$ \\
Present & $656(3)$ & $631(1)$ & $627(2)$ & $-27(-1)$ & $-4(1)$ \\
Absent &
\end{tabular}

Note. Cost $_{1}=$ mean for transfer block minus last training block; $\operatorname{Cost}_{2}=$ different color at transfer minus same color at transfer. Exp. = experiment. 
training now pressed . for red and / for green in transfer. If explicit color report in training produced associations between words and responses, opposite-color responses should be facilitated at transfer, relative to same-color responses, because the responses remain the same as in training. There should be no difference between opposite-color responses in transfer and target-present responses in the last training block, and there should be a large difference between same-color responses in transfer and target-present responses in the last training block.

If explicit color report in training produced associations between colors, words, and responses, then target-present responses should be longer in transfer than in the last training block. Moreover, there should be no difference in transfer between same- and opposite-color responses. Both effects should occur because the specific combinations of colors, words, and responses acquired in training do not repeat in transfer.

\section{Method}

Subjects. The subjects were 32 volunteers from the introductory psychology subject pool who were screened for red-green color blindness with the Ishihara (1987) test. None had served in Experiments 1-7.

Apparatus and stimuli. The apparatus and stimuli were the same as those used in the previous experiments. The response keys were slightly different, however. This experiment used $z, x$, period, and slash keys, which were the two leftmost and two rightmost keys on the bottom row of the standard QWERTY keyboard.

Procedure. The procedure was the same as Experiment 7, in that target color was reported in training and in transfer. There were 64 transfer trials, including both same-color and different-color word pairs (as in Experiments 5-7). In addition, the physical responses required to report target color and target absence were different in transfer than in training. Half of the subjects pressed the . key for red targets, the / key for green targets, and the $x$ key for nontargets during training. The other half pressed the $x$ key for red targets, the $z$ key for green targets, and the . key for nontargets during training. At transfer, each group changed mapping, reversing the keys they pressed to report target presence. Thus, the first group pressed / for red targets and for green targets, and the second group pressed $z$ for red targets and $x$ for green targets.

\section{Results}

Training. The mean reaction times and percentage of error scores for target-present and target-absent responses in the 16 training blocks are presented in Figure 8. As in Experiments 2, 4,6 , and 7 , reaction times were slower for target-present than for target-absent responses, and error rate was higher.

Reaction time and error rate decreased with practice, and the speedup in reaction time followed a power function. Power functions were fitted to the mean reaction times. The predicted values appear as solid lines in Figure 8; the points represent the observed values. Measures of goodness of fit and the parameters of power functions fitted to the mean reaction times appear in Table 2. The fits were good.

Reaction times and error rates were subjected to 2 (target present vs. absent) $\times 16$ (practice block) ANOVAs. The reaction time ANOVA found significant main effects of target

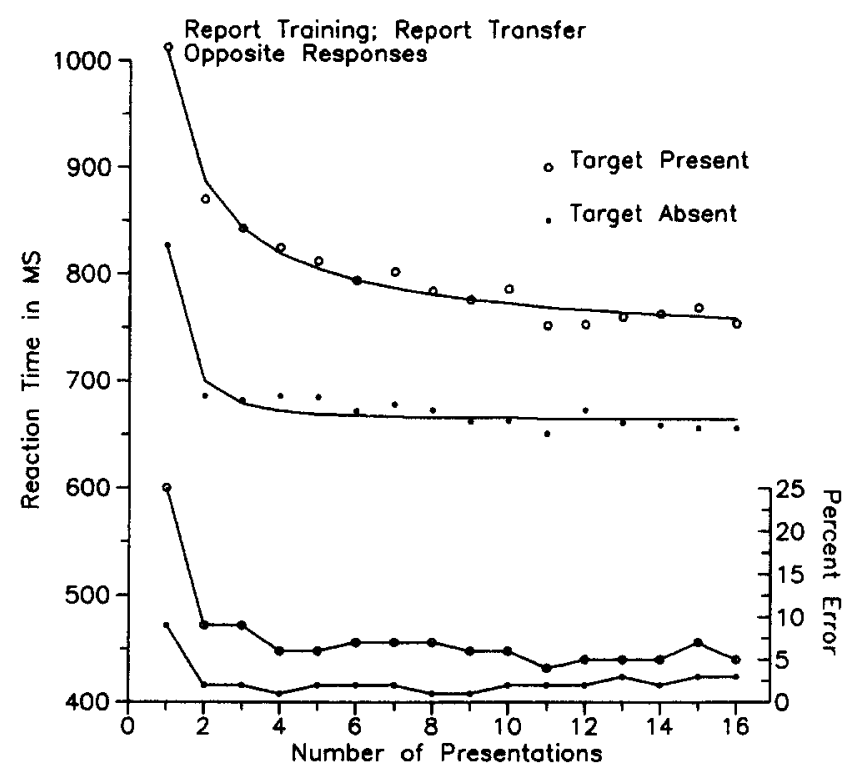

Figure 8. Mean reaction times (top two lines, left-hand $y$ axis) and error rates (bottom two lines, right-hand $y$ axis) for target-present (open circles) and target-absent (filled circles) responses from the training phase of Experiment 8 as a function of number of presentations (for the reaction times, the lines represent the best fitting power function, and the circles represent the observed data).

presence, $F(1,31)=112.81, M S E=34,972.58$, and practice block, $F(15,465)=34.00, M S E=5,185.45$, and a significant interaction between target presence and practice, $F(15,465)=$ 7.45, $M S E=1,969.32$. The error rate ANOVA found significant main effects of target presence, $F(1,31)=55.81, M S E=$ 114.04 , and practice block, $F(15,465)=17.28, M S E=40.63$, and a significant interaction between them, $F(15,465)=7.21$, $M S E=24.91$.

Transfer. The mean reaction times and error rates for the last training block and the transfer block are presented in Table 5. Subjects reported target color in training as well as in transfer. There was a large cost of changing the mapping between training and transfer for target-present responses. There was a small benefit for target-absent responses, perhaps because the mapping was the same in training and transfer. In the transfer block, reaction times were $15 \mathrm{~ms}$ faster for opposite-color target-present responses than for same-color target-present responses and $4 \mathrm{~ms}$ faster for opposite-color target-absent responses.

The mean reaction times and error rates from the transfer block were analyzed in 3 (presentation: last training trial vs. same-color vs. opposite-color transfer trials) $\times 2$ (target present vs. absent) ANOVAs on the mean reaction times and error rates. In the reaction time ANOVA, there was a significant interaction between presentation and target presence, $F(2,62)=15.75, M S E=2,516.37$, which we analyzed with planned comparisons. For target-present responses, in which the response mapping was reversed, reaction times were slower in the transfer block than in the last training block (813 ms vs. $754 \mathrm{~ms}$ ), $F(1,62)=29.01, M S E=2,516.37$. By contrast, for target-absent responses, in which the response mapping 
was not reversed, reaction times were faster in the transfer block than in the last training block ( $629 \mathrm{~ms}$ vs. $656 \mathrm{~ms}), F(1$, $62)=6.18, M S E=2,516.37$. Further planned comparisons revealed no difference between same- and opposite-color trials for target-present responses, $F(1,62)=1.43, M S E=2,516.37$, or for target-absent responses $(F<1.0)$.

The error rate ANOVA yielded similar results. The interaction between presentation and trial block was significant, $F(2$, $62)=4.24, M S E=26.37$, and planned comparisons revealed a significantly higher error rate in transfer than in the last training block for target-present responses ( $8 \%$ vs. $5 \%), F(1$, $62)=9.91, M S E=26.37$, but no significant difference for target-absent responses $(1 \%$ vs. $2 \% ; F<1.0)$. Further planned comparisons revealed no significant difference between sameand opposite-color trials for target-present or target-absent responses (both $F \mathrm{~s}<1.0$ ).

\section{Discussion}

The training data showed evidence of automatization, in that reaction time decreased as a power function of practice. The transfer data, by themselves and in contrast with the last training block, suggest that automatic performance may depend on an associative combination of color, word, and response information. If performance depended only on associations between words and responses, opposite-color targetpresent responses in transfer should have been as fast as target-present responses in the last training block because the same word-response pairings were repeated, and they should have been faster than same-color target-present responses in transfer because same-color transfer targets required a different response than in training. Neither of these effects was apparent in the data. Target-present responses were slower in transfer than in the last training block and no different for same- and opposite-colors in transfer because the trained combinations of colors, words, and responses did not not repeat at transfer.

\section{General Discussion}

The experiments suggested that information about target color was acquired during automatization but not expressed during automatic performance unless specific combinations of colors, words, and responses were repeated. Experiments 1 and 2 showed that color information was encoded during training on the focused-attention task because it was available to a retrieval task that assessed recognition memory for color-word pairings. The subsequent experiments showed that changing color at transfer had no effect on automatic performance unless the encoding and retrieval task both required explicit report of color (Experiments 4, 7, and 8). There was little sensitivity to color changes at transfer if explicit color report was required in transfer but not in training (Experiment 5 ), or if it was required in training but not in transfer (Experiment 6), or if it was not required in either training or transfer (Experiment 2). Experiment 7 suggested that the sensitivity to color change observed in Experiment 4 was due to associations between words and responses or colors, words, and responses rather than to associations between words and colors because subjects showed no sensitivity to color change when the response keys changed at transfer, even though the task required them to report color during training and transfer. Experiment 8 suggested that the sensitivity to color change observed in Experiment 4 was due to associations between colors, words, and responses rather than to words and responses because changing the color associated with a word and response slowed reaction times substantially. These data have implications for the attention hypothesis and for the nature of the representations that underlie performance in various retrieval tasks.

\section{The Attention Hypothesis}

The attention hypothesis predicts that the focused-attention orienting task should cause color information to be encoded during automatization because focusing attention on the target caused subjects to attend to the target's color. Experiments 1 and 2 confirmed this prediction, in which recognition memory was used as a retrieval task. The attention hypothesis did not predict whether color information would be expressed in automatic performance, but the results are nevertheless interpretable in terms of the attention hypothesis. The retrieval task in Experiments 3-8 emphasized speed and ease of access, and color information may have been retrieved too slowly or too infrequently to have much impact on the expression of automaticity. These results are important because they are among the first to support a distinction between what is encoded and what is retrieved in the automaticity literature (also see Logan, 1988, Experiment 5). They provide further support for the idea that automaticity is a memory phenomenon by showing that the distinction between encoding and retrieval, which has driven memory research for decades (e.g., Morris et al., 1977; Roediger, 1990; Tulving \& Thompson, 1973 ), is also important in automaticity.

The present experiments suggested that the results of target selection are stored in memory, which completes the analysis of the four kinds of attentional selection described by Treisman (1969). Previous data have suggested that the results of input selection (Logan \& Etherton, 1994) and analyzer selection (Logan, 1988, 1990) are encoded in memory during automatization, but the results of output or response selection are not (at least in the sense of storing associations with physical responses; Logan, 1990; see also Fisk \& Schneider, 1984; Shiffrin \& Schneider, 1977). The present Experiments 4, 7 , and 8 suggested that the results of output selection may be encoded in some cases, tied together with the results of other kinds of analyses. Together, these investigations support the attention hypothesis and encourage further investigation.

The attention hypothesis provides a basis for predicting what is learned during automatization and what is expressed in automatic performance. Learning can be predicted from a task analysis that reveals what people must attend to when they perform the task; people should learn about the things they attend to. The expression of learning in automatic performance can also be predicted from a task analysis that reveals what people attend to. The present experiments suggest that the task analysis should be extended to include the representations that people have in memory as a result of previous 
exposure to the task and an assessment of the availability of those representations to the retrieval process. Attention determines the cues that drive the retrieval process, and the requirement to respond quickly constrains the selection of relevant traces in memory. Traces that take a long time to be retrieved are unlikely to contribute much to automatic performance.

\section{What's in an Instance?}

The present data suggested that the representations acquired during automatization and the representations expressed during automatic performance do not represent all stimulus attributes uniformly. Instances are not "mental snapshots" that replicate the stimulus entirely (cf. Brown \& Carr, 1993; Carr, Brown, \& Charalambous, 1989). Instead, the attributes of the stimulus are filtered by attention, and only those that are relevant to the task are represented explicitly in the instance (see also Lassaline \& Logan, 1993; Logan, 1990; Logan \& Etherton, 1994).

The present experiments go beyond the previous ones in showing that some attributes may be encoded during automatization but not expressed during automatic performance. The idea that different retrieval tasks are sensitive to different stimulus attributes is a familiar one in the current literature on implicit and explicit memory (see, for example, Hintzman, 1990; Jacoby \& Brooks, 1984; Roediger, 1990), but it is relatively new in the automaticity literature. The idea is consistent with the instance theory of automaticity, which originally claimed that instances were processing episodes that represented traces of the various decisions the person engaged in while processing the stimulus (Logan, 1988, p. 495). Processing episodes have structure, and different parts of the structure may be important in encoding and retrieval.

The idea is also consistent with later elaborations of the theory that claimed that instance representations were propositional (Logan, 1990; Logan \& Etherton, 1994). Propositional representations can have a complex structure that is built by connecting elementary propositions that refer to the same arguments (Kintsch \& van Dijk, 1978). The complex representations can be retrieved in whole or in part by appropriate retrieval cues. The present experiments are consistent with this idea. Experiments 3, 5, and 6 suggested that automatic performance can be supported by propositions that do not include color as an argument, whereas Experiments 1 and 2 suggested that propositions about color are available in memory, even though they are not accessed during automatic performance. The experiments do not allow us to rule out alternatives to propositional representations, but they do suggest that, whatever their nature, the representations acquired during automatization have several parts that can be accessed separately and expressed differentially in performance on various retrieval tasks.

This is the first time that we have drawn a sharp distinction between different parts of an instance and proposed that different parts might be retrieved at different times. The formal development of the instance theory has assumed that all instances are the same and all instances are retrieved at the same time, following the same retrieval time distribution
(Logan, 1988, 1992b). Those assumptions were made primarily for convenience. It was much easier to prove that the outcome of the race followed a power function if the distribution of retrieval times was the same for each instance. However, Logan (1992b) showed that the power function proof would still hold if the distributions had the same shape (i.e., the same Weibull-distribution exponent), even if their means and standard deviations were different, and simulations showed that all parameters of the distribution could vary widely without compromising the power function proofs. Consequently, the idea we proposed here, that different parts of an instance take different amounts of time to be retrieved, need not compromise the power function predictions, which are the heart of the formal instance theory.

\section{Conclusions}

The present experiments supported a distinction between the contents of the memory trace and the parts of the trace that are expressed in a retrieval task. They suggested that attention determines what goes into a memory trace in encoding as well as what is taken out of it at retrieval time. What is encoded and what is retrieved are best understood in a task analysis that specifies what is attended during encoding and what is attended during retrieval. The experiments placed special emphasis on the accessibility of traces at retrieval time. Traces that are retrieved slowly or infrequently are likely to have little impact on the expression of automaticity, even though they were encoded during automatization.

\section{References}

Balota, D. A., \& Chumbley, J. I. (1984). Are lexical decisions a good measure of lexical access? Journal of Experimental Psychology: Human Perception and Performance, 10, 340-357.

Barsalou, L. W. (1995). Storage side effects: Studying processing to understand learning. In A. Ram \& D. Leake (Eds.), Goal-driven leaming (pp. 407-419). Cambridge, MA: MIT Press.

Battig, W. F., \& Montague, W. E. (1969). Category norms for verbal items in 56 categories: A replication and extension of the Connecticut category norms. Journal of Experimental Psychology, 80, 1-46.

Broadbent, D. E. (1958). Perception and communication. Elmsford, NY: Pergamon Press.

Brown, J. S., \& Carr, T. H. (1993). Limits on perceptual abstraction in reading: Asymmetric transfer between surface forms differing in typicality. Joumal of Experimental Psychology: Learning, Memory, and Cognition, 19, 1277-1296.

Carr, T. H., Brown, J. S., \& Charalambous, A. (1989). Repetition and reading: Perceptual encoding mechanisms are very abstract but not very interactive. Joumal of Experimental Psychology: Leaming, Memon, and Cognition, 15, 763-778.

Cohen, A., Ivry, R. I., \& Keele, S. W. (1990). Attention and structure in sequence learning. Joumal of Experimental Psychology: Learning, Memory, and Cognition, 16, 17-30.

Craik, F. I. M., \& Lockhart, R. S. (1972). Levels of processing: A framework for memory research. Journal of Verbal Learning and Verbal Behavior, 11, 671-676.

Etherton, J. L. (1992). Encoding of position and color during the acquisition of automaticity. Unpublished master's thesis, University of Illinois.

Fisk, A. D., \& Schneider, W. (1984). Consistent attending versus consistent responding in visual search: Task versus component 
consistency in automatic processing development. Bulletin of the Psychonomic Society, 22, 330-332.

Hintzman, D. L. (1990). Human learning and memory: Connections and dissociations. Annual Review of Psychology, 41, 109-139.

Ishihara, S. (1987). Ishihara's tests for colour-blindness. Tokyo: Kanehara \& $\mathrm{Co}$.

Jacoby, L. L., \& Brooks, L. R. (1984). Nonanalytic cognition: Memory, perception and concept learning. In G. H. Bower (Ed.), The psychology of leaming and motivation: Advances in research and theory (Vol. 18, pp. 1-47). New York: Academic Press.

Kahneman, D. (1973). Attention and effort. Englewood Cliffs, NJ: Prentice Hall.

Kahneman, D., Treisman, A., \& Burkell, J. (1983). The cost of visual filtering. Joumal of Experimental Psychology: Human Perception and Performance, 9, 510-522.

Kintsch, W., \& van Dijk, T. A. (1978). Toward a model of text comprehension and production. Psychological Review, 85, 363-394.

Kirsner, K., \& Dunn, J. (1985). The perceptual record: A common factor in repetition priming and attribute retention. In M. I. Posner \& O. S. Marin (Eds.), Attention and performance XI (pp. 547-565). Hillsdale, NJ: Erlbaum.

Kučera, H., \& Francis, W. N. (1967). Computational analysis of present-day American English. Providence, RI: Brown University Press.

Lassaline, M. L., \& Logan, G. D. (1993). Memory-based automaticity in the discrimination of visual numerosity. Joumal of Experimental Psychology: Leaming, Memory, and Cognition, 19, 561-581.

Logan, G. D. (1988). Toward an instance theory of automatization. Psychological Review, 95, 492-527.

Logan, G. D. (1990). Repetition priming and automaticity: Common underlying mechanisms? Cognitive Psychology, 22, 1-35.

Logan, G. D. (1992a). Attention and preattention in theories of automaticity. American Joumal of Psychology, 105, 317-339.

Logan, G. D. (1992b). Shapes of reaction time distributions and shapes of learning curves: A test of the instance theory of automaticity. Joumal of Experimental Psychology: Leaming, Memory, and Cognition, $18,883-914$.
Logan, G. D., \& Etherton, J. L. (1994). What is learned during automatization? The role of attention in constructing an instance. Joumal of Experimental Psychology: Learning, Memory, and Cognition, $20,1022-1050$.

Logan, G. D., \& Klapp, S. T. (1991). Automatizing alphabet arithmetic. I. Is extended practice necessary to produce automaticity? Joumal of Experimental Psychology: Leaming, Memory, and Cognition, 17, 179-195.

Morris, C. D., Bransford, J. D., \& Franks, J. J. (1977). Levels of processing versus transfer appropriate processing. Joumal of Verbal Learning and Verbal Behavior, 16, 519-533.

Newell, A., \& Rosenbloom, P. S. (1981). Mechanisms of skill acquisition and the law of practice. In J. R. Anderson (Ed.), Cognitive skills and their acquisition (pp. 1-55). Hillsdale, NJ: Erlbaum.

Nissen, M. J., \& Bullemer, P. (1987). Attentional requirements of learning: Evidence from performance measures. Cognitive Psychology, 19, 1-32.

Roediger, H. L., III. (1990). Implicit memory: Retention without remembering. American Psychologist, 45, 1043-1056.

Shiffrin, R. M., \& Schneider, W. (1977). Controlled and automatic human information processing: II. Perceptual learning, automatic attending, and a general theory. Psychological Review, 84, 127-190.

Stadler, M. A. (1995). The role of attention in implicit serial learning. Joumal of Experimental Psychology: Leaming, Memory, and Cognition, $21,674-685$.

Treisman, A. (1969). Strategies and models of selective attention. Psychological Review, 76, 282-299.

Treisman, A., Kahneman, D., \& Burkell, J. (1983). Perceptual objects and the cost of filtering. Perception \& Psychophysics, 33, 527-532.

Tulving, E., \& Thompson, D. M. (1973). Encoding specificity and retrieval processes in episodic memory. Psychological Review, 80, 352-373.

Uyeda, K. M., \& Mandler, G. (1980). Prototypicality norms for 28 semantic categories. Behavior Research, Methods, \& Instrumentation, $12,587-595$. 


\section{Appendix}

Words Used in the Experiments

\begin{tabular}{llll}
\hline Metals & Countries & Vegetables & Furniture \\
\hline Iron & France & Carrot & Chair \\
Copper & America & Peas & Table \\
Steel & Russia & Corn & Bed \\
Gold & England & Bean & Sofa \\
Aluminum & Germany & Lettuce & Desk \\
Silver & Canada & Spinach & Lamp \\
Tin & Italy & Asparagus & Couch \\
Zinc & Spain & Broccoli & Dresser \\
Brass & Mexico & Celery & Bureau \\
Lead & Ireland & Cabbage & Chest \\
Bronze & Japan & Cauliflower & Bookcase \\
Platinum & Sweden & Radishes & Cabinet \\
Nickel & Brazil & Potato & Davenport \\
Magnesium & Switzerland & Tomato & Footstool \\
Uranium & Norway & Cucumber & Buffet \\
Tungsten & Australia & Beets & Bench \\
\hline
\end{tabular}

Received February 15, 1995

Revision received March 7, 1995

Accepted March 13, 1995 